\title{
Developing Design Cracker Anacardium occidentale (mente) for Home Industrial
}

\author{
Dr. Ir. Soetyono Iskandar, \\ Mt., Mpd. (Nidn: 0015035403) \\ Fakultas Pendidikan Teknik Mesin Universitas Geri Makassar
}

\begin{abstract}
These purpose of my research was to design the peeled of cutting and made its quality and quantity of the cracker mente. The method for evaluation was designing and testing several peel models, testing crack effectively, and testing for its quality and quantity cracker. With developing design craker mente, we found the cracker home industrial. The cracker mente have developed design a cutting knife with follow the mente's the top of the ellipse knife. The length effectively was the maximum produce cracker quality is $8 \mathrm{~mm}$ with width mente average 25-27 mm. The result of the desain knife for cracker mente has to be grade mente quality to produce to be $79,25 \%$ each $1 \mathrm{~kg}$ with time peeling take 26 menit, 55 detik.
\end{abstract}

Kata kunci : Peeling mente.

\section{Introduction}

Mente is one of planted that has a high economic value at the national, and international market. Commodity horticulture at South Celebes enough much. Data from The Center of Statistic (CS) extension areal horticulture mente at South Celebes are 66.167,05 Ha, the total prodution reaching 23.369,92 tons. The regions that produce mente the great at South Celebes are Bone, Sidrap Pangkep, Barru, Sinjai, Bulukumba, and Selayar.

Agribusiness mente at South Celebes id makerting and preparation. For marketing at South Celebes could be to buy for its company. The farmer bargaining position with its value and have a profit for its working. Where the company efforted grade value for the farmer. Mente price is Rp. $5500 / \mathrm{kg}$ and grade mente is Rp. $45.000,-/ \mathrm{kg}$ overflow $100 \mathrm{~kg}$ mente produce $20 \mathrm{~kg}$ grade mete. So that efforted to be grade mente kernel we have income up than $50 \%$.

Processing cracker mente by company in scale home industrial at South Celebes commonly used tools kacip mente. The tool worked to put a knife up the mente and press down, so that the rind was peeled. And then split the mente, but the tool did not have a transform point at the center so that when spilt the mente seldom took off. After observation from the workers of the mete at region Barru got information total average mente that can be spilt in a day are 5-6 kg. Rismunandar (1986), capasity porduction mente used the tool kacip mente with power workers are $5-8 \mathrm{~kg} /$ day.

Capacity product information can find 50\% mente good and 50\% other ugly. And the good mente are very different with ugly mete. By survey at Supermarket Alfa Tamalanrea the price of ugly mete are $\mathrm{Rp}$ $58.000 / \mathrm{kg}$, and the good mente are $\mathrm{Rp} 61.875 / \mathrm{kg}$.

Azis (2008) has design the spilt of mente with transform system. These tool has grade its capacity production in $1 \mathrm{~kg}$ at $1 \mathrm{jam}$ and prosentase good mente was $74,45 \%$. Less of the tool was limited of the knife so that the small mete cannot spilt well.

For finding quality design kacip mete, indeed the knowledge about the engine must be notice all aspect of-kacip mente design.

Forces at kacip mente among other things were:

Massa tool components Kacip Mente

Relation with massa, volume and specific weight can be formula as the next :

$\mathbf{W}_{\mathbf{t}}=\mathbf{V} \times \mathbf{p}$

Explanation:

$\mathrm{W}_{\mathrm{t}}=$ massa tool component kacip mete $(\mathrm{kg})$

$\mathrm{V}=$ Volume component $\left(\mathrm{mm}^{3}\right)$

$\mathrm{P}=$ specific weight steel.

Forces Stick Press

(Khurmi 1985) to know how many transform forces stick press can be used formula as the next;

$\mathbf{F}=\mathbf{2 a} \times \mathbf{F}_{\mathrm{p}} / \mathbf{d c}$

Explanation:

$\mathrm{F}=$ Forces load from experiment mente $(\mathrm{N})$

$\mathrm{F}_{\mathrm{p}}=$ Force transform axle press $(\mathrm{N})$ 
$\mathrm{a}=$ length transform $\mathrm{arm} /$ axle press $(\mathrm{m})$

$\mathrm{dc}=$ Diameter Cam $(\mathrm{mm})$

Force effort endurance by axle press

(Khurmi 1985) formula for force can be efforted by axle press is :

$F=\frac{\pi^{2} E \quad I}{4 V(2 l)^{2}}$

Explanation :

$\mathrm{F}=$ Force effort load endurance by press axle $(\mathrm{kg})$

$\mathrm{E}=$ modulus elastic $\left(\mathrm{N} / \mathrm{mm}^{2}\right)$

$\mathrm{I}=$ moment inertia $\left(\mathrm{mm}^{4}\right), \mathrm{I}=\pi \mathrm{d}^{4} / 64$

$\mathrm{V}=$ coefisien pengaman.

Calculation Bench Strength

$l=$ high distance from axle at when loaded time. $(\mathrm{mm})$

For calculation bench strength can used formula as the next :

$\sigma_{\mathrm{t}}=\mathbf{F} / \mathrm{A}$

$F=\sigma_{t} \times A$

$A=\mathbf{p x} \mathbf{l}$

Explanation :

$\sigma_{\mathrm{t}}=$ tension pull bench $\left(\mathrm{N} / \mathrm{mm}^{2}\right)$

$\mathrm{F}=$ force at the bench $(\mathrm{N})$

$\mathrm{A}=$ surface bench $\left(\mathrm{mm}^{2}\right)$

$\mathrm{p}=$ length bench $(\mathrm{mm})$

1 = width bench $(\mathrm{mm})$

$>\quad$ Work for acting press

Formula for work is :

$\mathbf{W}=\mathbf{F}_{\mathrm{p}} \mathbf{x}$ a

Explanation :

$\mathrm{W}=$ Work (joule)

$\mathrm{F}_{\mathrm{p}}=$ force stick move press $(\mathrm{N})$

$\mathrm{a}=$ length arm stick (m)

$>\quad$ Forces tool Press

Power can be found by the tool press is :

$\mathbf{P}=\mathbf{W} / \mathbf{t}$

Explanation :

$\mathrm{P}=$ Power tool press (Watt)

$\mathrm{W}=$ Work (joule)

$\mathrm{t}=$ time for pressing (second)

$>\quad$ Bolt Axle Press

For calculating bolt axle press used formula :

$\sigma_{\mathrm{t}}=\mathbf{F} / \mathrm{A}$

$\mathrm{A}=\boldsymbol{\pi} \mathrm{d}_{\mathrm{i}}^{2} / 4$

Explanation:

$\sigma_{\mathrm{t}}=$ tension pull/press $\left(\mathrm{N} / \mathrm{mm}^{2}\right)$

$\mathrm{F}=$ force work at the bolt $(\mathrm{N})$

$A=$ surface bolt $\left(\mathrm{mm}^{2}\right)$

$\mathrm{d}_{\mathrm{i}}=$ diameter center/critis bolt $(\mathrm{mm})$

$>\quad$ Press Plat

For appointment central as the next :

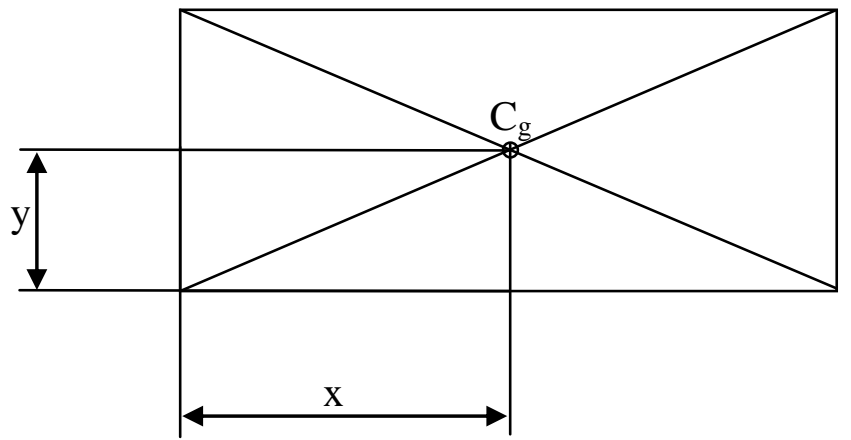


Gambar 2.2. Press Plat

Explanation :

$\mathrm{C}_{\mathrm{g}}=$ central gravity

$\mathrm{X}=$ distance central to horizontal edge

$\mathrm{y}=$ distance central to vertical edge

$>\quad$ Strength setting weld plat

For calculating weld strength used formula :

$\sigma_{t}=$ F/A

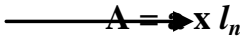

Explanation ;

$\sigma_{\mathrm{t}}=$ tension pull/press $\quad$ occour $\left(\mathrm{N} / \mathrm{mm}^{2}\right)$

$l$ = length the weld $(\mathrm{mm})$

$\mathrm{t}=$ thick plat $(\mathrm{mm})$

$\mathrm{a}=$ thick weld $(\mathrm{mm})$

$l_{n}=$ length efektively $(\mathrm{mm})(\ln =l-2 \mathrm{a})$

$>\quad$ Load and Tension Move Spiral

For calculating spiral used formula as the next :

$\mathbf{C}=\mathbf{D} / \mathbf{d}$

$\mathrm{C}=$ index spiral

Explanation :

$\mathrm{D}=$ diameter everage wrap spiral $(\mathrm{mm})$

$\mathrm{d}=$ diameter wire spiral $(\mathrm{mm})$

$K=\frac{4 c-1}{4 C-4}+\frac{0,615}{C}$

Explanation :

$\mathrm{K}=$ Factor tension from Wahl (number Wahl)

$\delta=\frac{8 W D^{3} n}{G d^{4}} \longrightarrow \mathrm{N}=\mathrm{n}+(1,5$ sampai 2$)$

$W=\frac{\delta G d^{4}}{8 D^{3} n}$

Explanation :

$\mathrm{W}=\operatorname{load}(\mathrm{N})$

$\delta=\operatorname{deflection}(\mathrm{mm})$

$\mathrm{G}=$ Modulus move

$\mathrm{N}=$ total wrap spiral

$\mathrm{n}=$ total actively wrap

$T_{g} \frac{K .8 W C}{\pi d^{2}}$

Explanation :

$\mathrm{T}_{\mathrm{g}}=$ tension move $\left(\mathrm{N} / \mathrm{mm}^{2}\right)$

Bench

For counting the bench moment needed to know bent moment, bent tension and tension maximum, (Khurmi 1985 ) endurance bent moment and maximum tension can used formula as the next :

$\mathbf{W}_{\mathrm{b}}=\mathbf{b h}^{2} / 6$

Explanation :

$\mathrm{W}_{\mathrm{b}}=$ moment bent endurance $\left(\mathrm{mm}^{3}\right)$

$\mathrm{b}=$ length bench $(\mathrm{mm})$

$\mathrm{h}=$ width bench $(\mathrm{mm})$

$\tau_{\max } \frac{M_{\max }}{W_{b}}$

Explanation :

$\tau_{\max }=$ tension bent maximum $\left(\mathrm{N} / \mathrm{mm}^{2}\right)$

$\mathrm{M}_{\max }=$ moment bent maximum $(\mathrm{N} / \mathrm{mm})$, can be found from analysis forces was worked again the bench with Free Body Diagram. 


\section{Method Research}

This research was conducted at the workshop mechanical engineering Universitas Negeri Makassar with in research for 6 months. The tools this research used were saw machine, frais machine, lathe machine, weld machine, sney, tapped, screw-driver, tools, weight, and stopwatch. Materials the research used are steel plat, galvanis pipe, axle, stainless-steel knife, plat strep, nut, and bolt. The prototype peel machine as the shown picture 2.1

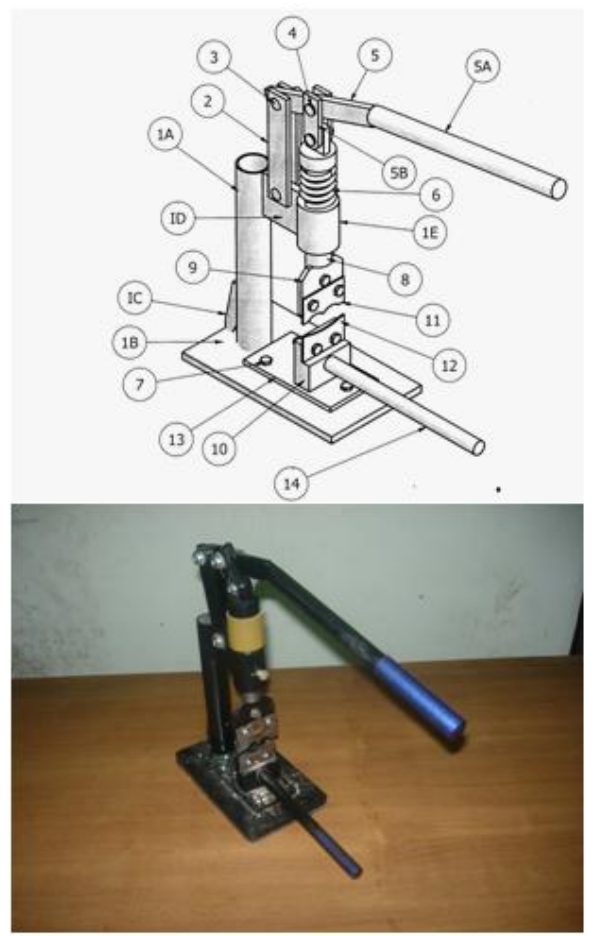

Gambar. 2.1 Result prototype "Peel Machine Mente".

\subsection{Result}

\section{Result And Discussion}

\section{A. Calculation Result Hasil}

a. Force occur at the knife

Mente can be peeled if found press force/ loaded. The load was press force from stick press, axle press, arm and knife can be calculated with data meanwhile ;

Dimension of mente : length $\mathrm{x}$ wideth $\mathrm{x}$ high $=35 \times 17 \times 25 \mathrm{~mm}$

Experiment press of mente got force for peeling mente is $500 \mathrm{~N}$ and length of the knife $=55 \mathrm{~mm}$.

F. $a-R_{B} . L=0$

$$
F \cdot \frac{l}{2}-R_{b} \cdot L=0
$$

$R_{b}=\frac{F \cdot L / 2}{L}$

$\mathrm{R}_{\mathrm{B}}=\frac{F}{2}$

$\mathrm{R}_{\mathrm{B}}=\frac{500}{2}=250 \mathrm{~N}$

$\sum F_{v}=0$

$\mathrm{R}_{\mathrm{A}}+\mathrm{R}_{\mathrm{B}}-\mathrm{F}=0$

$\mathrm{R}_{\mathrm{A}}=\mathrm{F}-\mathrm{R}_{\mathrm{B}}$

$\mathrm{R}_{\mathrm{A}}=500-250=250 \mathrm{~N}$

$\mathrm{M}_{\mathrm{C}}=\mathrm{R}_{\mathrm{B}} \times 55 / 2$

$\mathrm{M}_{\mathrm{C}}=250 \times 22,5=5625 \mathrm{Nmm}=5,625 \mathrm{Nm}$ 


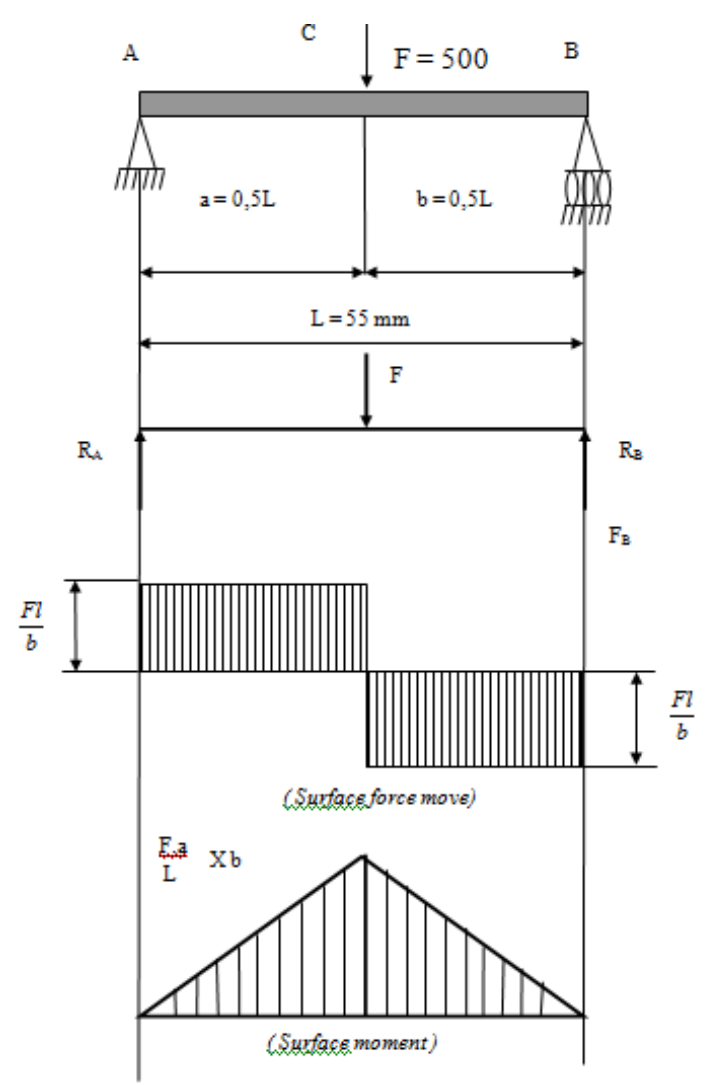

Picture 4.1 . Diagram force move and moment of the knife when process press the mente. $\sum M_{A}=0$

Moment maximum occur at point $\mathrm{C}$

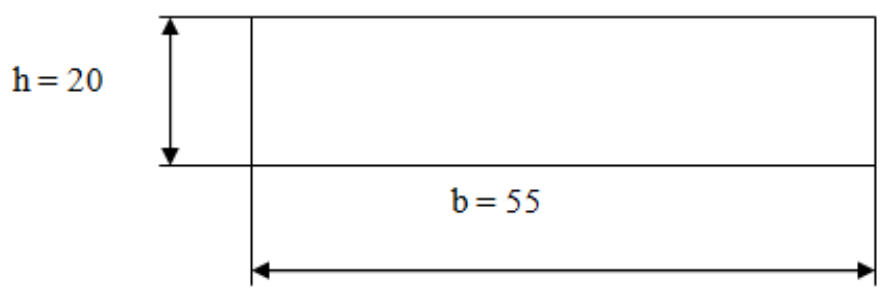

Picture 4. 2. Moment maximum at point C

Moment bent endurance, $\mathrm{W}_{\mathrm{b}}=\frac{b h^{2}}{6}=\frac{55 x(20)^{2}}{6}=3666,7 \mathrm{~mm}^{3}$

Tension maximum, $\boldsymbol{\sigma}_{\max }=\frac{M_{\max }}{W_{B}}=\frac{5625}{3666,7}=1,5 \mathrm{~N} / \mathrm{mm}^{2}$

Tension bent $=3 / 4$ tension pull, because used material ST. 42 with tension pull maximum $420 \mathrm{~N} / \mathrm{mm}^{2}$, so tension bent maximum $=3 / 4420=315 \mathrm{~N} / \mathrm{mm}^{2}$

Tension bent allowance, $\sigma_{b}=\frac{\sigma_{b \max }}{v}, \mathrm{v}=$ safety factor, taken 6 .

$$
\begin{aligned}
\boldsymbol{\sigma}_{\mathrm{b}} & =\boldsymbol{\sigma}_{\mathrm{b} \max } / 6 \\
& =315 / 6=52,5 \mathrm{~N} / \mathrm{mm}^{2}
\end{aligned}
$$

Calculation found value tension bent allowance more than tension bent maximum $\left(\boldsymbol{\sigma}_{\mathrm{b}}>\boldsymbol{\sigma}_{\mathrm{b} \text { max }}\right)$ at the bench. Mean the knife able to endurance the load occured without to be bent. 


\section{b. Force Stick Press}

$\mathbf{F}=\mathbf{2} \mathbf{a} \times \mathbf{F t}_{\mathrm{p}} / \mathbf{d}$

By experiment force pressat the mente found force press is $500 \mathrm{~N}$

Explanation :

$\mathrm{F}=$ Force from experiment with used test machine pull/press ( Galdabini ), when the tested can be seen at enclosure.

$\mathrm{Ft}_{\mathrm{p}}=$ Force stick press $(\mathrm{N})$

$\mathrm{a}=$ Length stick press $=250 \mathrm{~mm}$

$\mathrm{dp}=$ Diameter stick press $=20 \mathrm{~mm}$

$\mathrm{Ft}_{\mathrm{p}}=(500 \times 20) /(2 \times 250)=20 \mathrm{~N}=2 \mathrm{Kg}$

c. Work to treat the Pressure

$\mathbf{W}=\mathrm{Ft}_{\mathrm{P}} \mathbf{x}$ a

Where :

$\mathrm{W}=$ Work (joule)

$\mathrm{Ft}_{\mathrm{P}}=$ Force stick press $(\mathrm{N})$

$\mathrm{a}=$ distance transfer force $=5 \mathrm{~mm}=0,05 \mathrm{~m}$ ( assumption)

$\mathrm{W}=20.0,05=1 \mathrm{Nm}$ atau 1 Joule

d. Power Kacip Mente

$\mathbf{P}=\mathbf{W} / \mathbf{t}$

Where:

$\mathrm{P}=$ power tool press 0,2 watt.

$\mathrm{W}=\mathrm{Work}=0,2$ Joule

$\mathrm{t}=$ time $/$ time press (second)

e. Force able to endurance by axle press

$\mathbf{F}=\frac{\pi^{2} E I}{4 V(2 l)^{2}}$

Where :

$$
\begin{aligned}
& \mathrm{F}=\text { Force }(\mathrm{kg}) \\
& \mathrm{E}=\text { Modulus Elastic }\left(\mathrm{N} / \mathrm{mm}^{2}\right) \\
& \mathrm{I}=\text { Moment inertia }\left(\mathrm{mm}^{4}\right) \\
& V=\text { Coefisien safety for steel 3-5 }
\end{aligned}
$$

$1=$ High distance from axle at load $(\mathrm{mm})$

$\mathrm{d}=$ Diameter axle press $(\mathrm{mm})$

$$
\begin{aligned}
& \mathbf{I}=\pi \mathbf{d}^{4} / \mathbf{6 4}=3,14 \times(35)^{4} / 64=73624,4 \mathrm{~mm}^{4} \\
& \mathbf{F}=\frac{\pi^{2} E I}{4 V(2 l)^{2}} \\
& \mathrm{~F}=\frac{(3,14)^{2} \times 210.10^{3} \times 73624,4}{4 \times 5 \times(2 \times 180)^{2}}=23524,76 \mathrm{~N}=2352,5 \mathrm{Kg} .
\end{aligned}
$$

Therefore, axle press safefor received loaded $500 \mathrm{~N}$, because load maximum can be ebdurance axle press is $23524,7 \mathrm{~N}$ atau $2352,5 \mathrm{Kg}$

$$
\begin{aligned}
& \text { f. Calculation Bolt Strength Axle Press } \\
& \sigma_{\mathrm{t}}=\mathrm{F} / \mathrm{A} \quad \mathrm{A}=\pi \mathrm{d}_{\mathrm{i}}^{2} / 4 \\
& \sigma_{\mathrm{t}}=\frac{4 F}{\pi d_{i}^{2}}=\frac{4 \times 500}{3,14(8)^{2}}=9,96 \mathrm{~N} / \mathrm{mm}^{2} \\
& \sigma_{\mathrm{t} \text { izin }}=\mathrm{F}_{\mathrm{t} \text { izin }} / \mathrm{A} \quad \mathrm{A}=\pi \mathrm{d}_{\mathrm{i}}^{2} / 4 \\
& \sigma_{\mathrm{t} \text { izin }}=\frac{4 F_{\text {tizin }}}{\pi d_{i}^{2}}=\frac{4 \times 21000}{3,14(8)^{2}}=418,3 \mathrm{~N} / \mathrm{mm}^{2}
\end{aligned}
$$


Because tension pull bolt more than small from tension pull allowance for bolt M8 $\left(\sigma_{\mathrm{t}}<\sigma_{\mathrm{t}}\right.$ allow), so bolt was safe and was not damage.

\section{g. Calculation Strength Weld at the Plat Scaffold \\ 1. Gusset}

$\mathbf{F}=\mathbf{A} \cdot \boldsymbol{\sigma}_{\mathbf{l}}$

Where :

$\sigma_{\mathrm{t}}=$ Tension in elektroda/wire weld $=42 \mathrm{Kg} / \mathrm{cm}^{2}$.

$F=$ Force $(\mathrm{Kg})$

$\mathrm{A}=$ Surface horizontal result weld $\left(\mathrm{cm}^{2}\right)$

$\mathrm{a}=$ Thick weld $=0,6 \mathrm{~cm}$.

$l=$ Length result weld $=1 \mathrm{~cm}$.

Therefore

$\mathbf{a}=1 / 2 \sqrt{2} \quad$ (because angle weld $45^{\circ}$ or same with $\sin 45$ )

$=0.5 \times 1,4=0,7 \mathrm{~cm}$

$\mathbf{A}=\mathbf{2}$. a . $\mathbf{l}$ (because has 2 result weld, therefore times 2)

$$
=2 \cdot 0,7 \cdot 0,1=0,14 \mathrm{~cm}^{2}
$$

Angle $\alpha$ as tension at the surface weld is $45^{\circ}$, mean occurt tension moved.

At the table $\alpha$ so that :

$\alpha=45^{\circ} \longrightarrow \boldsymbol{\tau}_{\mathbf{t}}=\mathbf{0 , 8 5}$ jadi $\tau_{\mathrm{l}}=0,85 . \tau_{\mathrm{t}}=0.85 .42=35,7 \mathrm{Kg} / \mathrm{cm}^{2}$

Therefore, $\mathrm{F}=\mathrm{A} \cdot \tau_{1}=0,14.35,7=4,99 \mathrm{Kg}$ or $5 \mathrm{Kg}$

So that, force can be received by result weld $5 \mathbf{K g}$ as force must be received by result weld $\mathbf{4 , 9} \mathbf{K g}$ (massa total components kacip at page 19). Mean the result weld was safe.

\section{Column}

$\mathbf{F}=\mathbf{A} \cdot \sigma_{1} \longrightarrow \mathbf{A}=\mathbf{a} \cdot \mathbf{l}$

where:

$\sigma_{\mathrm{t}}=$ Tension in elektroda/wire weld $\left(\mathrm{Kg} / \mathrm{cm}^{2}\right)$

$F=$ Force $(\mathrm{Kg})$

$\mathrm{A}=$ Surface horizontal result weld $\left(\mathrm{cm}^{2}\right)$

$\mathrm{a}=$ Thick weld $(\mathrm{mm})$

$l=$ Length result weld $(\mathrm{mm})$

data :

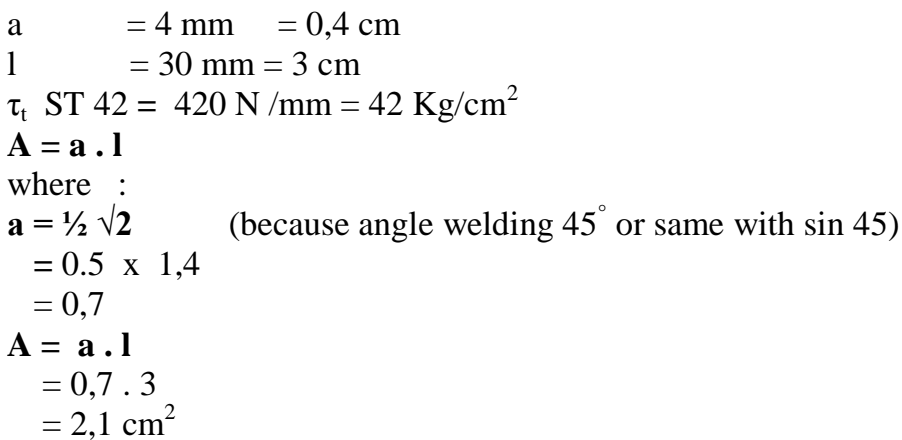

Angle $\alpha$ as a tension at surface weld is $45^{\circ}$, an then here is occur moved tension.

At the table $\alpha$ for :

$\alpha=45^{\circ} \longrightarrow \boldsymbol{\tau}_{\mathbf{t}}=\mathbf{0 , 8 5}$ jadi $\boldsymbol{\tau}_{\mathrm{l}}=0,85 . \tau_{\mathrm{t}}$

$$
\begin{aligned}
& =0.85 .42 \\
& =35,7 \mathrm{Kg} / \mathrm{cm}^{2}
\end{aligned}
$$

Teherefore, $\mathbf{F}=\mathbf{A} \cdot \boldsymbol{\tau}_{1}$

$$
=2,1 \cdot 35,7
$$$$
=74,97 \mathrm{Kg}
$$

Therefore, loaded can be received by result weld is $74,97 \mathbf{K g}$ whereas loaded has to received by result weld 2,8 Kg. This mean result weld at the plat is safe.

\section{h. Calculation Load and Tension Moved Spiral}

Data :

Total wrap $(\mathrm{N})$

Length free

$$
\begin{aligned}
& =12 \\
& =100 \mathrm{~mm} \\
& =30 \mathrm{~mm} \\
& =2 \mathrm{~mm}
\end{aligned}
$$

Diameter wire spiral $(\mathrm{d})$ 
Modulus move $(\mathrm{G})$

The length $(\delta)$

Solution :

$\mathrm{C}=\mathrm{D} / \mathrm{d}=\frac{30}{2}=15$

$\mathrm{K}=\frac{4 C-1}{4 C-4}+\frac{0,615}{0,9}=\frac{4(15)-1}{4(15)-4}+\frac{0,615}{015}$

$\mathrm{K}=\frac{259}{56}+\frac{0,615}{15}=\frac{919,44}{840}=1,09$

$\mathrm{N}=\mathrm{n}+(1,5$ till 2$) ;$ taken 2

$\mathrm{n}=\mathrm{N}-2=12-2=10$

whereas loaded can be enduranced by spiral when pressed

$$
\begin{aligned}
& \delta=\frac{8 W D^{3} n}{G d^{4}} \quad \mathrm{~W}=\frac{\delta G d^{4}}{8 D^{3} n} \\
& \mathrm{~W}=\frac{112 x\left(83.10^{3}\right) x(2)^{4}}{8 x(30)^{3} \times 12}=\frac{148736000}{2592000}=57,3 \mathrm{~N} \\
& \tau_{\mathrm{g}}=\frac{K .8 W C}{\pi d^{2}}=\frac{1,09 x 8 \times 57,3 \times 15}{3,14 x(2)^{2}}=\frac{7494,84}{12,56}=596,72 \mathrm{~N} / \mathrm{mm}^{2}
\end{aligned}
$$

Whereas loaded can be endurance by spiral is :

$\mathrm{Wa}=$ massa Axle press + massa Arm + massa + tension press

$\mathrm{Wa}=0,56+0,24+2(\mathrm{Kg})$

$\mathrm{Wa}=2,8 \mathrm{~kg}$

Calculation has load of the spiral $(\mathrm{Wa})=2,8 \mathrm{Kg}$, maximum loaded can enduranced by spiral $(\mathrm{W})=57,3 \mathrm{Kg}$. Therefore $\mathrm{W}>\mathrm{Wa}$, so the spiral and used safely.

\section{B. Result Maker}

Specification tool peeling mente has made can be seen at Table 4.1 berikut;

Table 4.1. Spesification tool peeling mente.

\begin{tabular}{|l|l|}
\hline Material & ST-42 \\
Dimension Length & $220 \mathrm{~mm}$ \\
Dimension Wideth & $120 \mathrm{~mm}$ \\
Dimension High & $330 \mathrm{~mm}$ \\
\hline Material Knife & Steinless Steel \\
Dimension top knife & $53 \times 35 \times 1,2 \mathrm{~mm} 53 \times 35 \times 1,2$ \\
Dimension bottom knife & $\mathrm{mm}$ \\
\hline Force Press & $2 \mathrm{Kg}$. \\
\hline
\end{tabular}

\section{Result Experiment}

Process experiment has designed with cutting knife, was experimental quality and quantity peeling.

\section{Result design experiment cutting knife}

Indicated dimension mente come to the data as ; 1 . Length $=32-35 \mathrm{~mm}, 2$. Wideth $=25-27 \mathrm{~mm}, 3$. thick $=16$ $-19 \mathrm{~mm}, 4$. Radius at the back $=25 \mathrm{~mm}, 5$. Radius at the front $=6 \& 8 \mathrm{~mm}$. Result design experiment cutting knife can be seen at table 4.2 as the next. 
Table 4.2. Result experiment 3 model cutting knife.

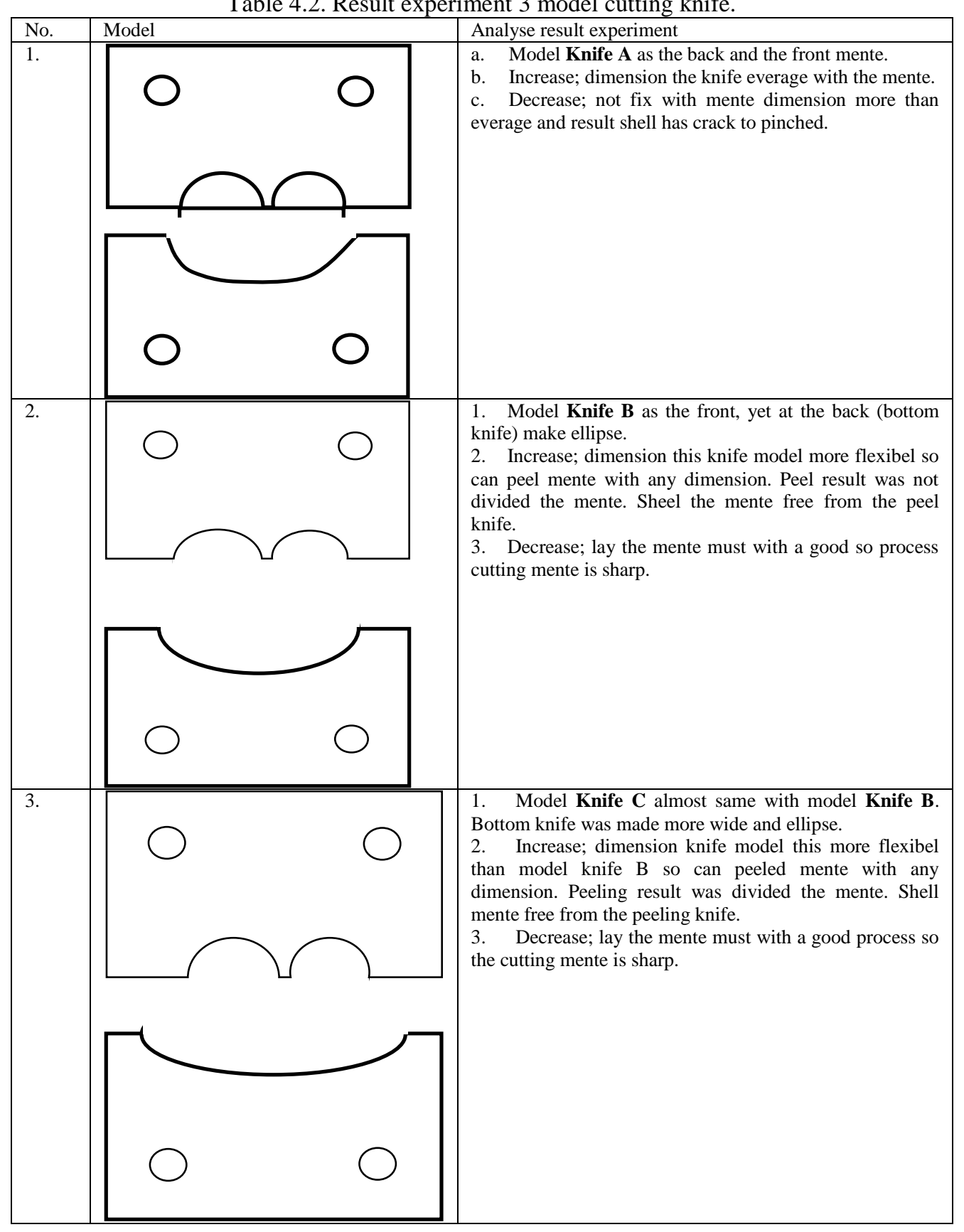

From this result experiment data hasil pengujian, so model knife B was used in process the next experiment, yet from the processing maker model knife $\mathrm{C}$ more easy to work because more wide its ellipse.

\section{Experiment result cutting knife}

Experiment result cutting knife experiment cutting knife for to know effective length in peeling the mente so result peeling was not for cutting mente. The fist data from distance top knife and bottom knife $=24$ $\mathrm{mm}$. Distance top knife and bottom knife at will be cut $=14 \mathrm{~mm}$ (calculate $0 \mathrm{~mm}$ step). Result experiment length step cutting knife can be seen at table 4.3 berikut. 
Table 4.3. Result experiment length step cutting knife.

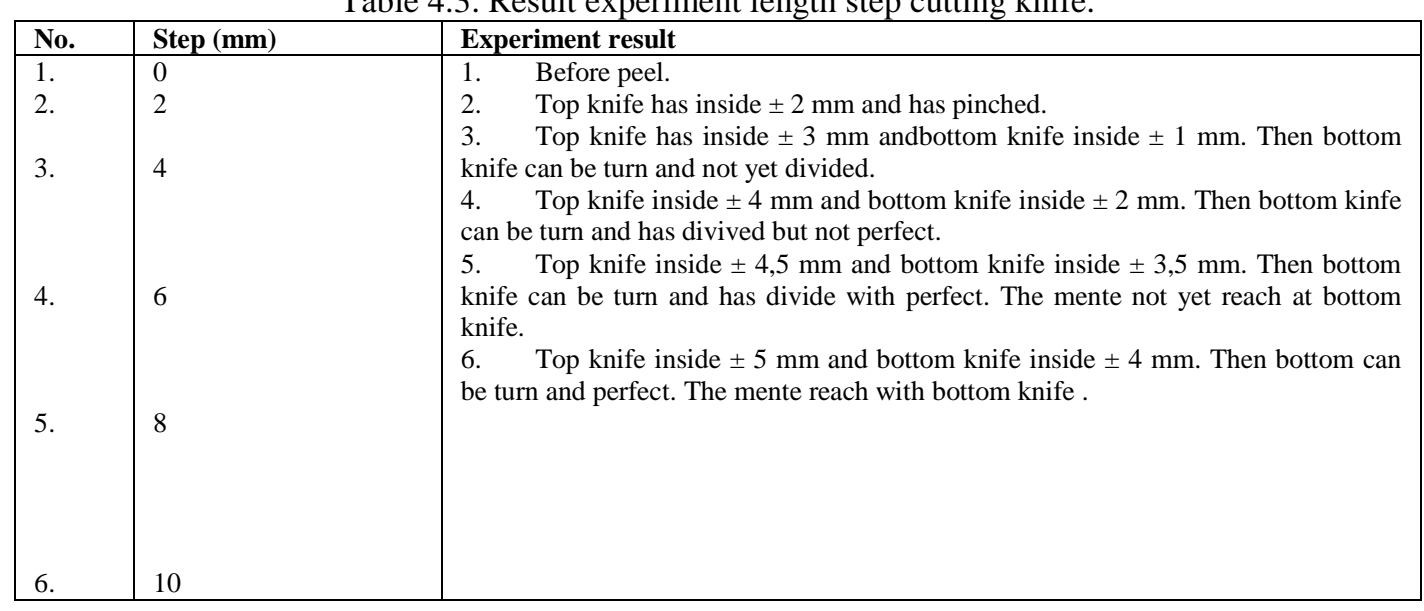

Experiment result again step peel gave a good peel at inside in $8 \mathrm{~mm}$.

\section{Quality and quantity experiment result peel}

The quality and quantity purpose experiment at tool peel mente was to know capacity of the tool.. Data the quality and quantity experiment can be seen at tabel 4.4 as the next..

Table 4.4. Data quality and quantity experiment peeling mente.

\begin{tabular}{|l|l|l|l|l|}
\hline No. & Total in 1Kg & Time (minute, second) & Entire & Divided \\
\hline 1 & 175.0 & $29^{\prime}, 35^{\prime \prime}$ & 130 & 45 \\
\hline 2 & 172.0 & $27^{\prime}, 15^{\prime \prime}$ & 137 & 35 \\
\hline 3 & 173.0 & $25^{\prime}, 20^{\prime \prime}$ & 140 & 33 \\
\hline 4 & 174.0 & $25^{\prime}, 30^{\prime \prime}$ & 143 & 31 \\
\hline Average & 173.5 & $26^{\prime}, 55^{\prime \prime}$ & 137.5 & 36 \\
\hline & & Percentage & $79.25 \%$ & $20.75 \%$ \\
\hline
\end{tabular}

\section{Discussion}

Developing Design Cracker Anacardium occidentale (mente) for Home Industrial with specification data it is moveable and practice for peeling the mente.

Repaired design of cutting knife with increased capacity and quality. See table 4.4. Tool experiment result can shown the need everage time for peeling mente eachs $1 \mathrm{Kg}$ is 26 minute, 55 second. Time was needed for peeling mente is better than Azis's maked is 47 minute 11 second. If an hour the tool capacity could peeled 2 $\mathrm{Kg} /$ hour.

Increased in quality peeling is $79,25 \%$ mente the entire and 20,75 mente divided. Has increased in quality peeling than the tool by Azis was, 74,45\% mente entire. Yet, from the produce target with divided mente was $20,75 \%$ its result enough many in decrease. When the experiment has done to peel it, has not dried so the mente still at its shell. But this research has to continue to know how much the temperature and the exact time for drying for quality increased result peeling.

\section{Conclution And Rec0mmendation}

From this experiment and discussion its conclusion as the next:

Peeling mente has development design with the top of the ellipse knife. The effectively length has a quality product, maximum peeling was $8 \mathrm{~mm}$ with width mente average $25-27 \mathrm{~mm}$. The result tool design peeling mente has increased an quality peeling mente to be $79,25 \%$ for $1 \mathrm{~kg}$ with peeling time in 26 minute, 55 second. Developing Design Cracker Anacardium occidentale (mente) for Home Industrial has done at workshop with our students. We hope the produce could be continued to community by socialization and experimental. But this research has to continue to know how much the temperature and the exact time for drying for quality increased result peeling.

\section{Greeting}

Thank you very much to Rector State University Makassar and Head of Research for giving me an opportunity to do my research. 


\section{Reference}

[1] ------. 2003. Statistik Pertanian Tanaman Pangan \& Hortikultura Sulawesi Selatan. BPS Prop. Sul-Sel. Makassar.

[2] Nieman, Gustav. 1992. Elemen Mesin, Jilid I. Erlangga. Jakarta.

[3] Khurmi, Gustav. 1985. Machine Design. Eurasia Publishing House. New Delhi.

[4] Rukmana, Rahmat. 1995. Budidaya dan Pengolahan Pasca Panen Bawang Merah. Kanisius. Yogyakarta.

[5] Singgih,Wibowo. 1992. Budidaya Tanaman Bawang Merah. Aneka Ilmu. Semarang.

[6] Sugiharto. 1992. Budidaya dan Pengolahan Pasca Panen Bawang Merah. Kanisius. Yogyakarta.

[7] Sularso. 1987. dasar Perancangan dan Pemilihn Elemen Mesin. PT. Pradnya Paramita. Jakarta. 